\title{
Social Navigation Research Agenda
}

\author{
Andreas Dieberger \\ IBM Almaden Research Center \\ 650 Harry Rd, San Jose, CA 95120, USA \\ +1(408) 9271470 \\ andreasd@us.ibm.com
}

\author{
Kristina Höök, Martin Svensson, Peter Lönnqvist \\ HUMLE, Swedish Institute for Computer Science \\ Box 1263, SE-164 29 Kista, Sweden \\ +4686331500 \\ kia@sics.se
}

\begin{abstract}
Social navigation ( $\mathrm{SN}$ ) emerged as a marriage between one-user-one-system scenarios and CSCW. It is a design approach based on either visualizing traces of other users' activities or on direct or indirect communication between users, with the goal to facilitate locating and evaluating information. Social Navigation has wide-ranging benefits, from social filtering over improving trust in eCommerce all the way to improving the user experience in general. However, as it is a new field many design issues are not properly researched yet. In this paper we outline a possible research agenda for the social navigation field, pointing out areas of social navigation in need of research initiatives.
\end{abstract}

\section{Keywords}

Social navigation, social filtering, trust, adaptive systems

\section{INTRODUCTION}

Social navigation [1] emerged out the observation that, although humans are very social beings, many of our systems do not support social interaction well. Recently, as recommender systems, social filtering and similar applications gain popularity, this trend seems to change. Still, SN systems are exceptions, not the rule.

As a design approach social navigation tries to raise awareness that social activities should be part of our information processing environments. The likely benefits are manifold, however, there are many unresolved and unresearched issues. We outline a research agenda for SN.

\section{FORMS OF SOCIAL NAVIGATION}

Social navigation may occur in a variety of ways. To date it is unclear what forms of SN are most beneficial for what uses. We can distinguish between systems where actors are co-present and their activities are immediately perceived others and systems where $\mathrm{SN}$ information is visible only as the aggregated history of previous, nonconcurrent, usage.

We also can distinguish between systems where actors are in direct contact with one another (direct social navigation), and systems where contact is anonymous and indirect (indirect social navigation). Very likely, all these forms have their justification, but each of them brings up a number of possible social issues.

\section{Availability of Social Navigation Information}

Some researchers propose including SN in every aspect of a computer system. Based on information on peer activities such a system could provide recommendations how to set a network mask, which printer in the office are most reliable, all the way to where to filing expense reports.

A contrasting view bases SN's utility on the task domain. Therefore SN functionality should not be built into every system by default. This view is especially concerned about leading users astray and about accountability issues.

Yet another approach looks for spatial metaphors as enablers for social navigation, drawing on work in architecture and urban design. These disciplines have a long tradition of observing and influencing how people navigate and socially interact, which would make spatial metaphors a good foundation for SN systems.

Note that this is not a complete list and that these approaches do not necessarily preclude each other. Clearly, research is needed to clarify which approach works best for which domains.

\section{RECOMMENDATIONS AND REPUTATIONS}

Many store-based recommender systems, such as Amazon.com, base recommendations people's purchases, because voting with a credit card is a strong statement indeed. However, these systems do not capture what happened before a purchase: did customers compare many items or did they know exactly what they wanted? Did they come back over and over again till making a decision? Similarly, there is little post-purchase feedback whether an item met a customer's needs.

Post-purchase information often can be found in advice and rating sites, like Epinions.com. An issue with these sites is that people need to be very happy or unhappy about a purchase before bothering to write a review, which often leaves out middle ground reviews, unless special incentives are given to provide reviews.

\section{Quality}

Reviews vary in quality. Sometimes reviewers themselves are rated to indicate whether they are "expert reviewers" or not. But is an "expert reviewer" a "domain expert" or somebody who likes to criticize products? Should somebody who became expert reviewer for travel guides be an expert reviewer for her first digital camera review?

Reputation management tries to approach some of these issues. Auction services, like eBay.com, were among the 
first to incorporate reputation information for buyers and sellers. Using reputations can solve some of the issues outlined earlier. However, recommendations need to be associated with individuals, essentially eliminating all anonymity provided by statistical approaches, characteristic for indirect social navigation. Voting or writing recommendations requires a deliberate effort. Therefore these systems operate based on possibly higher quality, but sparser information than systems using information on page views or purchases, collected as effortless by-products of the user activities. Most reputation and recommendation systems are susceptible to artificially inflated recommendations, which brings up the issue of trust.

\section{Trust}

Trust has become a hot issue lately; indeed trust might be the key issue deciding between success or failure of eCommerce. As long as the quality of recommendations, and the competence of reviewers cannot be guaranteed, it will be difficult to get people to fully trust SN systems. This is one of the areas where indirect social navigation provides a clear advantage, because information is aggregated over many people.

\section{Timeliness}

As indirect $\mathrm{SN}$ requires a lot of input, it is slow to adapt to changes in user preferences. This brings up issues of timeliness. An example is a forest path, which exists only as long as it is regularly used. Related metaphors could be used to indicate popular event Web sites like for the Olympic games. Such a site rapidly drops in popularity after the event, but the SN system will continue to rate the site as popular. By continually misleading people to visit the site, the popularity stays higher than it should be, permeating the problem.

\section{OTHER RESEARCH ISSUES}

The issues outlined above are clearly areas that require a lot more research. Unfortunately, defining quality and relevance can be even more complex, because the context can entirely redefine quality. In certain situations, high quality information (recommendations) might be ignored, because decision makers - for whatever reason - prefer to use different information sources. Harper [4] found that information workers at the International Monetary Funds, deemed relevant and correct information as uninteresting only because the people with power were not acting upon it. Such "frameworks of relevance" show that quality clearly is a very complex concept.

\section{Social Affordances and Awareness}

Systems based on social navigation concepts typically impact users' behavior. History-enriched environments can make people more aware of each other and thus contribute to a more social experience of the information space. When entering new applications, new services or interaction spaces, people need help getting started both on the system itself, as well as on the social conventions of the place.

Visible actions of other people can inform us of appropriate behavior and thus provide social affordances.
At the same time, awareness of others and their actions makes a space feel more alive and turn it into something we might perceive as place [2].

Such systems might be concerned primarily with improving the user experience, which has an impact on how much we trust people we meet, whether we would engage in commercial transactions with them or whether we would follow their recommendations. These issues could impact the design of chat rooms for customer support and online sales and - ultimately - how we do business online.

\section{Use Reshapes Experience}

Social navigation design can involve altering the structure of a space, like when recommender systems, such as Amazon.com, change the structure of their navigational space by creating links to related items. Such systems are a first step towards empowering actors to make the functionality and structure of a system, drift and to make our information spaces more fluid, ultimately empowering a user community to customize systems to their needs. Customization of this kind might jeopardize consistency in structure.

\section{Design issues}

To make social navigation systems work, SN cues need to be understandable and designs must appropriately convey how our individual actions will in turn influence the system. SN will require a certain, limited amount of visibility of our actions, which infringes on our privacy. Privacy concerns are a major stumbling block towards acceptance of SN systems. Erickson and Kellogg [3] use the concept of social translucence to describe that it is not only necessary to see other actors, but to clearly communicate that what information is disclosing and how it is used. Social translucence entails a balance of visibility, awareness of others, and accountability.

\section{SUMMARY}

Social Navigation might have a big impact on what future online interaction spaces will look like. However, there are many unresolved issues. There is a growing awareness of some of these issues, like trust and quality of recommendations. Others still require a lot of work. We outlined the key issues in the hope to inspire research initiatives in these areas.

\section{References}

1. Dieberger, A., et. al. Social Navigation: Techniques for Building More Usable Systems, interactions, 7, 6, 2000, 36-45.

2. Dourish, P. and Chalmers, M. Running out of Space: Models of Information Navigation, HCI'94, 1994.

3. Erickson, T. and Kellogg, W.A. Social Translucence: An Approach to Designing Systems that Support Social Processes, Trans. on HCI, 7, 1, 2000, 59-83.

4. Harper, R.H.R. Information that Counts: A Sociological View of Information Navigation, in Munro, A.J., Höök, K., and Benyon, D. (Eds.). Social Navigation of Information Space. Springer, London, 1999, 80-89. 